\title{
Multi-Domain Monitoring of Marine Environments using a Heterogeneous Robot Team
}

\author{
Florian Shkurti $^{1}$, Anqi Xu ${ }^{1}$, Malika Meghjani ${ }^{1}$, Juan Camilo Gamboa Higuera ${ }^{1}$, \\ Yogesh Girdhar ${ }^{1}$, Philippe Giguère ${ }^{2}$, Bir Bikram Dey ${ }^{1}$, Jimmy Li ${ }^{1}$, Arnold Kalmbach ${ }^{1}$, \\ Chris Prahacs ${ }^{1}$, Katrine Turgeon ${ }^{3}$, Ioannis Rekleitis ${ }^{1}$, and Gregory Dudek ${ }^{1}$.
}

\begin{abstract}
In this paper we describe a heterogeneous multirobot system for assisting scientists in environmental monitoring tasks, such as the inspection of marine ecosystems. This team of robots is comprised of a fixed-wing aerial vehicle, an autonomous airboat, and an agile legged underwater robot. These robots interact with off-site scientists and operate in a hierarchical structure to autonomously collect visual footage of interesting underwater regions, from multiple scales and mediums. We discuss organizational and scheduling complexities associated with multi-robot experiments in a field robotics setting. We also present results from our field trials, where we demonstrated the use of this heterogeneous robot team to achieve multi-domain monitoring of coral reefs, based on realtime interaction with a remotely-located marine biologist.
\end{abstract}

\section{INTRODUCTION}

We present a multi-robot system developed for environmental monitoring and surveying, which operates in the aerial, water surface, and underwater domains. Our primary objective is to explore the practicality of using autonomous robots to perform environmental monitoring, for instance by helping marine scientists conduct repeated inspections in a consistent, efficient, and comprehensive manner. To this end, our heterogeneous robot team interacts with human experts remotely to identify areas of interest based on live aerial feedback, and then autonomously collects visual footage of these areas at different scales and from multiple domains.

We are especially motivated to help marine biologists survey the long-term health of coral reefs. Coral reefs are extremely precious ecosystems: they occupy less than $0.1 \%$ of the world's ocean surface, yet they provide a habitat for $25 \%$ of all marine species [1]. Unfortunately, coral reefs have been decaying at an alarming rate in recent decades [2], and there has been a long-standing need among scientific communities to identify methods for their preservation [3].

Coral reefs are monitored conventionally by scientists, who must dive to reef sites on a regular basis to inspect their health visually. This approach is laborious and slow, since the scientists have to travel potentially large distances between live coral sites. A common complementary strategy

\footnotetext{
${ }^{1}$ School of Computer Science, McGill University fflorian, anqixu, malika, gamboa, yogesh, birbikram, jimmyli, akalmbach, cprahacs, yiannis, dudek\} ecim.mcgill.ca

${ }^{2}$ Département d'informatique et de génie logiciel, Université Laval philippe.gigueredift.ulaval.ca

${ }^{3}$ Department of Integrative Biology, University of Guelph kturgeon@uoguelph.ca
}

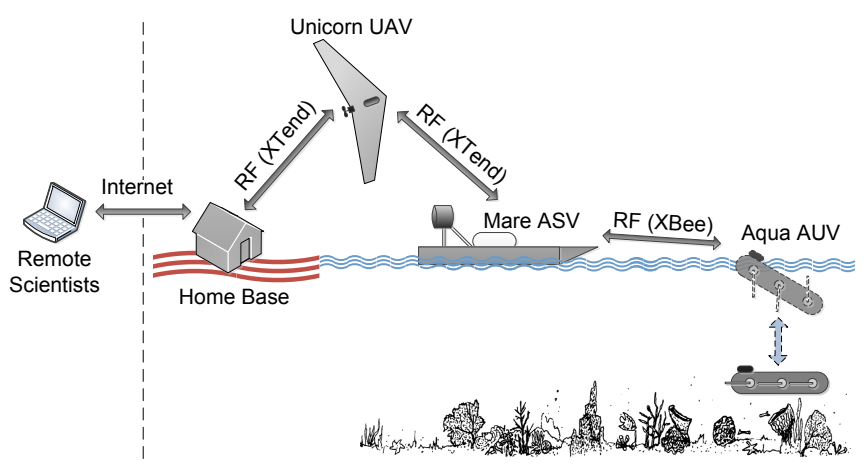

Fig. 1. Layout of our heterogeneous multi-robot system used for the monitoring of marine environments in collaboration with remote scientists.

is to plan each dive beforehand by identifying potentially rich reef patches based on aerial footage [3], although its effectiveness depends on having up-to-date satellite imagery with sufficient visual resolution and clarity.

We address the drawbacks of conventional reef monitoring methods by automating the data collection process using a team of robots, which is comprised of an Unmanned Aerial Vehicle (UAV), an Autonomous Surface Vehicle (ASV), and an Autonomous Underwater Vehicle (AUV). During a typical monitoring session, our UAV continuously collects aerial footage of a reef region and relays them to scientists via the Internet. Based on this live footage, the scientists then suggest sites for further inspection to the team of robots, which subsequently coordinate with each other to autonomously collect visual footage of these target regions. Our system thus provides comprehensive visual coverage of the specified reef sites, both from a broad aerial view and a close-up underwater view. This autonomous robotic team also significantly improves the efficiency of the monitoring process, by shifting the scientists' focus from laborious data collection to the selection of survey regions.

This team of robots operates in a top-down hierarchical structure that arises due to the diversity in each vehicle's operational range, and the need to communicate with remote scientists and the home base. This hierarchy, depicted in Fig. 1, is also beneficial to the monitoring process: the UAV can obtain coarse visual coverage of the entire reef region quickly, which allows the ASV and AUV to then focus on inspecting the most potentially interesting sub-regions at a finer resolution, while minimizing the time spent traveling over less important regions. 
This paper presents the first results where a remote expert (marine biologist) guides in real time a team of heterogeneous robots operating in air, sea surface and underwater, to inspect the health of a coral reef. Over 18 kilometers of flight and 1.5 kilometers of underwater traverses validated the robustness of our approach.

In this paper we will elaborate on individual components in this integrated multi-robot system, along with other important aspects of the marine monitoring tasksuch as scheduling and coordination of various roles occupied by the human and robot participants. We then discuss our field trial results, which demonstrates the efficiency of this multi-robot system for marine biologists to study coral reefs.

\section{RELATED WORK}

Our work aims to coordinate robots operating in diverse domains, to obtain multi-resolution imagery of marine environments. This is related to a number of works involving the planning and control of heterogeneous multi-robot systems.

Valdivia y Alvarado et al. [4] deployed a team of robots consisting of a micro aerial robot, an ASV, and an AUV, to perform monitoring of algal blooms in coastal environments. The structure and heterogeneity of their robotic team is very similar to ours, although our setup also incorporates realtime input from remote scientists, to provide more accurate identification of potential regions of interest.

Sujit et al. [5] studied the problem of coordinating multiple AUVs with a single UAV in a simulated grid world, where the AUVs followed paths generated by the UAV. The proposed path routing algorithm imposed constraints on the UAV's path to allow each AUV to surface and exchange information when the UAV flies over it. They evaluated the routing algorithm in simulation to demonstrate correctness, while ignoring pragmatic concerns such as weather conditions, hardware limitations and software failures.

Shafer et al. [6] developed a system in which a torpedo AUV and an autonomous kayak collaborated in search and coverage tasks such as underwater mine clearance. They demonstrated empirically that the heterogeneity of their autonomous vehicles can be exploited to optimize energy and time resources. Dunbabin et al. [7] proposed an algorithm for transporting data between a set of underwater sensor nodes, by having an AUV move over all the sensor nodes sequentially while minimizing its positional error. They also incorporated a surfacing behavior for the AUV to refine its positional estimates while traversing long distances, which is similar to the porpoising behavior proposed in our work.

Hsieh et al. [8] deployed multiple UAVs and ground vehicles to locate and track human targets in large indoors and outdoors environments using a Simultaneous Localization and Mapping (SLAM) approach. Their two-phase search process consists of identifying targets visually within a map of the target region, which is constructed from aerial footage obtained using UAVs. Subsequently, a human operator issues terrestrial vehicles to visually monitor and track the identified targets autonomously.

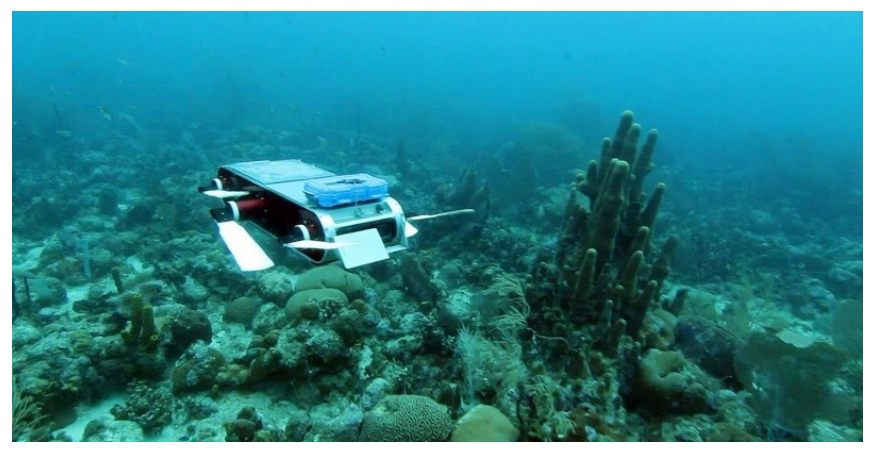

Fig. 2. The Aqua AUV navigating over a coral reef.

The systems described in [9], [10], [11], [12] are motivated by the application of robotics systems to environmental monitoring. In particular, the focus of these works lies in tracking oceanographic features, such as algal blooms, marine life and water conditions, and enabling scientists to interpret them. Das et al. [9] demonstrated a system for tracking varied oceanographic features, by coordinating an underwater glider with a surface drifter and satellite data. The system built by Podnar et al. [10] applies various levels of autonomy to coordinate ASVs and a UAV, for the purpose of data collection and monitoring by remote experts. Our work shares a similar motivation, which is to assist biologists at monitoring coral reefs by gathering multi-domain footage using a team of heterogeneous autonomous robots.

\section{HETEROGENEOUS MULTI-ROBOT SYSTEM FOR MARINE MONITORING}

We have developed a multi-robot system that is capable of autonomously collecting multi-domain imagery of specific sites of interest. Our system incorporates three vehicles that operate in diverse domains: the Unicorn fixed-wing $\mathrm{UAV}^{1}$, a catamaran-style ASV called the Marine Autonomous Robotic Explorer (MARE) [13], and the Aqua AUV platform [14].

This multi-robot system interacts with remotely-located biologists who are responsible for selecting local areas of interest for more comprehensive and up-close inspection. We facilitate this task by providing live visual coverage of the broad marine region, captured by our aerial robot, to the remote experts. Once our robots receive the scientists' directives in the form of waypoints at the start of each session, they then autonomously carry out the monitoring task by fulfilling a number of distinct roles:

- The Unicorn UAV is responsible for performing coverage of the entire survey region and provide up-to-date large-scale aerial imagery to the remote human scientists. After obtaining the expert-selected inspection sites, the UAV then re-broadcasts these waypoint directives to the other two robots underneath, while continuing to collect updated aerial footage of the entire region.

- The MARE ASV serves two primary roles: it is used to cache waypoint directives received from the Unicorn $\mathrm{UAV}$, and it also relays these messages to the Aqua

${ }^{1}$ From Procerus Technologies, www.procerusuav.com 
AUV when it surfaces. These roles are needed because the UAV has limited battery life, making it unable to wait until the Aqua UAV surfaces.

- The Aqua AUV is responsible for gathering fine-scale imagery by performing close-up inspection of the target sites. While Aqua mainly operates underwater to navigate to these sites and collect footage, it also regularly ascends to the surface to listen for further messages from MARE and to update its localization using GPS.

This team of autonomous robots carries out the monitoring task while communicating in an asynchronous manner. We will describe the functionalities of each robot in the following sections.

\section{A. Aqua $A U V$}

The Aqua AUV [14], as shown in Fig. 2, is a six-legged amphibious robot that can both swim underwater and walk on land. It maneuvers in water by actuating its six flippers, and its aluminum shell is designed to operate at depths up to $40 \mathrm{~m}$. It is powered by high-capacity Lithium-Ion batteries, and can operate under full load for more than five hours underwater.

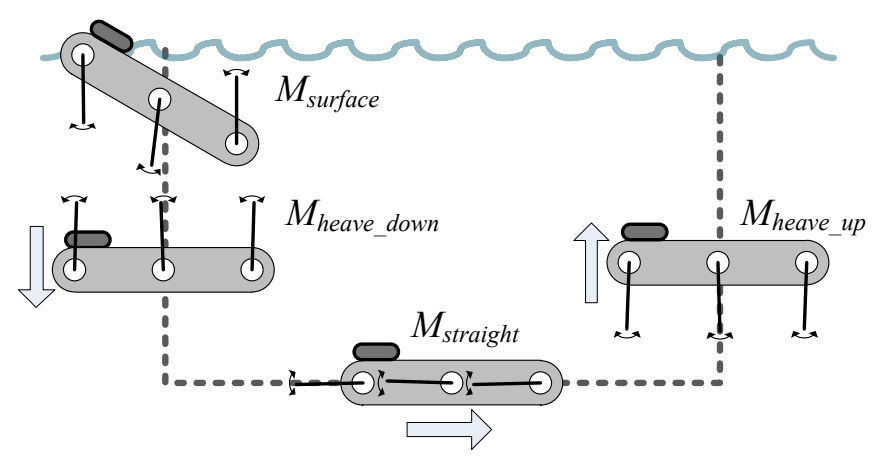

Fig. 3. The four states of the porpoising motion for the Aqua AUV.

1) Sensors: The Aqua AUV is equipped with a variety of sensors within its waterproof shell, which includes: three CMOS cameras, an inertial measurement unit (IMU), a pressure-based depth sensor, and an $\mathrm{XBee}$ digital radio transceiver. We also equipped the AUV with an externallymounted sensor kit to facilitate wireless communications, since the AUV's metallic shell highly attenuates the transmission of radio signals. This sensor kit contains a GPS unit and an XBee module, and augments Aqua's sensing capabilities by relaying GPS data and messages from the MARE ASV using the XBee communications link. Sensor processing and high-level planning is carried out by an embedded computer running Linux on a Core 2 Duo CPU, which interacts with another computer that regulates the six leg motors in realtime under the QNX OS.

2) Control and Porpoising Motion: Aqua's motion is regulated by an autopilot that allows both attitude control and depth control based on the IMU and depth sensor readings. For the purposes of our marine monitoring task, we deployed a GPS-based waypoint follower on top of the autopilot. This navigation system iteratively implements the following sequence of actions: ascend to the surface, collect GPS position estimates, descend to a fixed depth, adjust bearing towards current waypoint, and move forward for a given amount of time.

The combination of ascending, descending, and forward movement constitutes the porpoising motion of the Aqua robot. This navigation strategy was chosen to address several fundamental challenges imposed by the underwater environment on existing long-range localization algorithms. For instance, vision-based techniques for position estimation must cope with the possibility of severely limited underwater visibility, whereas acoustic localization methods would need to address multipath interference issues when operating in shallow underwater environments. We therefore chose the porpoising motion and GPS localization for increased reliability. To enable this motion, we configured the autopilot to implement the following three modes:

- $M_{\text {straight }}$ : straight forward motion at a fixed depth;

- Meave : controlled heave for ascent and descent; and

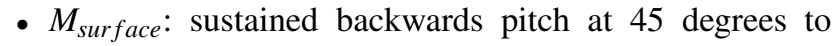
elevate the external sensor kit above the sea surface.

The autopilot implements proportional $(P)$ controllers for the pitch and roll axes, and a proportional-derivative $(P D)$ controller for the yaw axis. The latter derivative gain is essential to counteract the slow response time of this vehicle about the yaw axis [15].

While executing $M_{\text {straight }}$, the autopilot maintains a straight trajectory along a fixed heading angle at constant depth by regulating the roll angle about $0^{\circ}$ and the pitch angle based on the target depth. The $M_{\text {heave }}$ and $M_{\text {surface }}$ modes rely on a hovering gait described in [16], in which the roll and pitch gains are downscaled from $M_{\text {straight }}$ by a factor of ten to minimize oscillatory behaviors.

Since sea water is conductive, it is infeasible to receive GPS signals underwater. This was corroborated through empirical studies where we determined that less than $1 \mathrm{~cm}$ of seawater over a $12 \mathrm{~cm}^{2}$ patch GPS antenna is sufficient to preclude GPS reception Thus, Aqua's body must be inclined backwards to elevate the GPS antenna above the water surface. The $M_{\text {surface }}$ mode implements this behavior by combining a target pitch angle of $45^{\circ}$ with an upwards heave command. In this mode, Aqua's four back flippers are oriented downwards and oscillate with a large amplitude, while its two front flippers are pointed upwards with minimal oscillation. This results in both a net positive force and a net moment, which counteracts forces due to the back of the robot being raised above the water surface.

3) Software: We implemented a modular ROS-based software architecture for the Aqua robot to facilitate its highlevel control functionalities. One critical module in this ROS abstraction layer is a vision-based dialog [17] that allows a diver to communicate with Aqua directly using ARToolKit [18] fiducial markers. This interface allows us to easily reconfigure system parameters and experiment settings in the field with minimal overhead. 


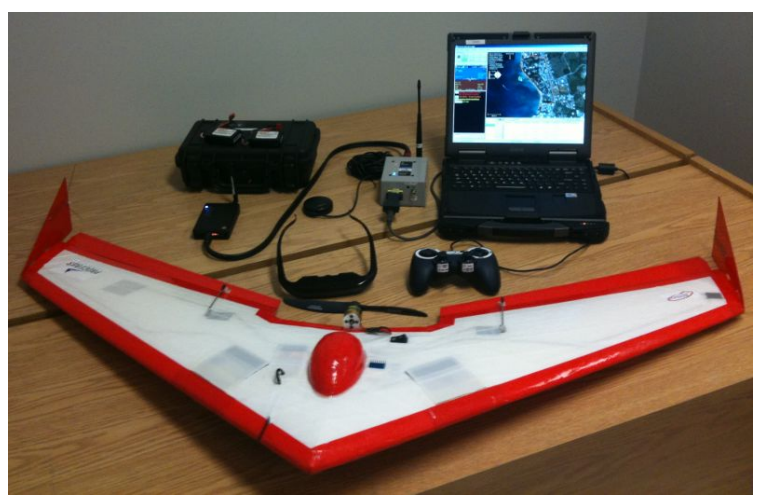

Fig. 4. The Unicorn is a fixed-wing UAV with an on-board autopilot microprocessor and a gimbal-mounted camera.

\section{B. Unicorn $U A V$}

The Unicorn UAV is a kite-sized fixed-wing aerial vehicle. This robot has a $1 \mathrm{~m}$ wingspan and is built from expanded polypropylene (EPP) foam, which is used to absorb and dissipate the collision force during touchdown. The Unicorn's brushless motor is powered by Lithium Polymer batteries, which allows the vehicle to operate at average ground speeds of $14 \mathrm{~m} / \mathrm{s}$ for up to 30 minutes of flight time.

1) Sensors: This UAV is equipped with multiple sensors, including an IMU, a GPS, and pressure-based altitude and speed sensors. These devices are integrated with the onboard autopilot micro-processor, which uses them to navigate autonomously based on waypoint directives issued from the home base. Communication between the autopilot and the home base is established via radio frequency using a highpower XTend modem; this allows the UAV to be controlled at multi-kilometer ranges. The Unicorn is also equipped with a CCD camera mounted on a pan-tilt gimbal, which allows the home base to receive live aerial feed through an analog radio frequency channel.

2) Software Infrastructure: In order to automate the aerial data collection process, we developed an application that interfaces with the commercial control software for the Unicorn UAV, and in particular incorporates an autonomous navigation controller for conducting complete and repeated aerial coverage [19]. In addition, a front-end interface allows a human operator to monitor the UAV's status, select useful aerial images to send to biologists for inspection, and validate waypoints chosen by the biologists before re-transmitting them to our team of robots.

\section{MARE ASV}

The Marine Autonomous Robotic Explorer (MARE) [13] is a robotic airboat developed to explore coral reefs and shallow seabeds. Its open catamaran, twin-pontoon design provides sufficient hydrodynamic stability to operate in turbulent open water environments. This vehicle is actuated using air propellers in a differential drive configuration, which is preferred over water-based propulsion mechanisms as it causes less underwater disturbance to shallow reefs. MARE's motors are powered by Lithium Polymer batteries, which provides over two hours of continuous operations.

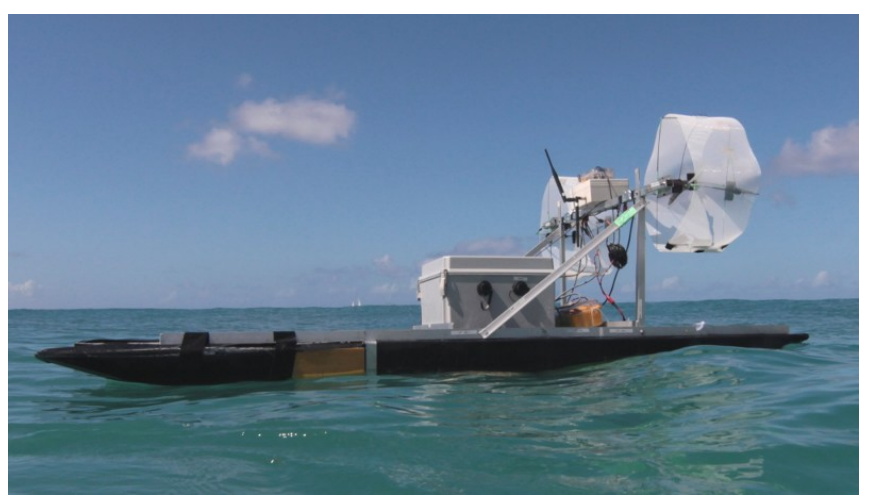

Fig. 5. The Marine Autonomous Robotic Explorer (MARE) is a catamaran robotic airboat that can operate in turbulent open water environments.

1) Sensors: Most of the electronics on-board are stored inside a large water-proof enclosure at the center of the chassis. The primary computing unit is a netbook computer that is responsible for interfacing with the motor micro-controller, powering and collecting data from sensors, and managing high-level autonomous behaviors. MARE is a vision-centric platform and is equipped with a high-definition downwardfacing camera. It also uses an IMU and a GPS device to track its location and pose in the water. Furthermore, MARE is capable of communicating over a variety of channels, including a WiFi link used to stream video, low-power XBee and long-range XTend radio transceivers used to relay information between the Unicorn UAV and Aqua AUV, and an analog transceiver that enables manual tele-operation at multi-kilometer distances.

2) Software Infrastructure: MARE uses a modular software architecture built using the ROS middleware. We have previously developed a number of autonomous navigation controllers for the MARE ASV [13], although we configured MARE to act as a stationary surface platform for relaying waypoint messages from the Unicorn UAV to the Aqua AUV.

\section{Interaction with Marine Scientists}

This multi-robot marine monitoring system incorporates input from marine biologists, who are responsible for identifying marine sites that are worth inspecting at a finer scale, based on the coarse-scale aerial footage captured by our UAV. These scientists, however, are not responsible for direct tele-operation control over any of the robots; robot navigation and the data collection process are both carried out autonomously. We developed a web interface that allows scientists off-site to remotely monitor aerial images obtained by our UAV and mark points of interest on individual images. These coordinates are transmitted as target GPS waypoints to the home base, and then broadcasted sequentially to the Unicorn UAV, to the MARE ASV, and finally to the Aqua AUV. This web interface allows scientists to interact with our robot team within the visual task domain while concealing underlying communication and control aspects, to ensure a smooth and intuitive user experience. 


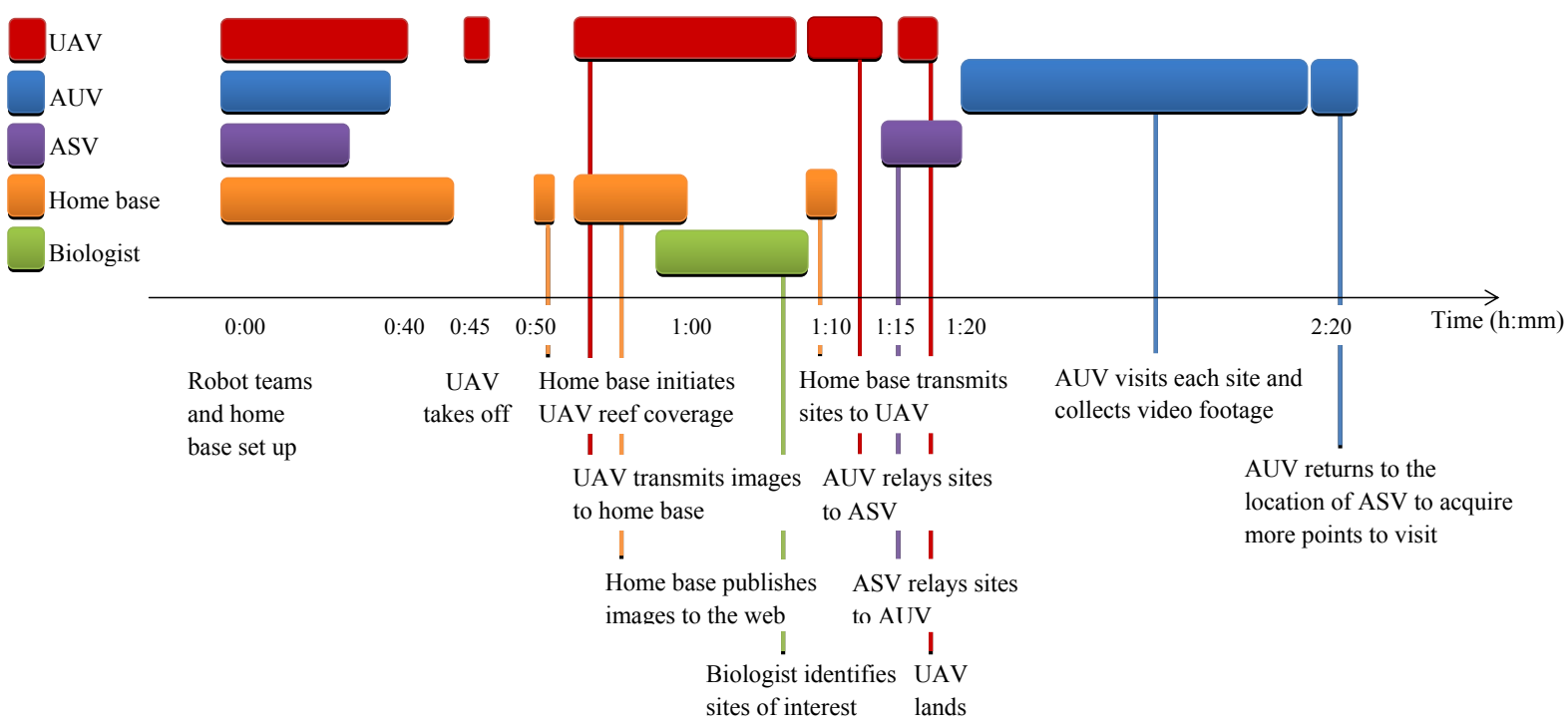

Fig. 6. Event timeline for our muli-robot coral reef monitoring sessions, lasting 2 hours and 30 minutes on average. Note: timeline is not to scale.

\section{CORAL REEF MONITORING TRIALS}

We conducted an extensive field trial comprised of multiple coral reef monitoring sessions, to evaluate the feasibility of our approach and to optimize system parameters. Fig. 6 depicts a simplified timeline for each monitoring session, starting with the launch of the Unicorn UAV and ending with the Aqua AUV visiting and recording underwater footage at a number of sites of interest within a tropical reef region. These sites were chosen by a biologist located about $4,000 \mathrm{~km}$ away from our reef region, who interacted with our system in real time through a web interface.

Each monitoring session lasted over 2 hours long, though a significant portion of that time was devoted to setting up the various robots at distinct operational sites, both on land and at sea. Each vehicle is supported by a small team of roboticists with the exclusive purpose of passively monitoring their operations during each session. Furthermore, given the complexity of communications arising from the nature of the experiment, a home base team was deemed necessary. Members of the home base team were responsible for monitoring the timing of the entire experiment and informing other teams of event progressions.

\section{A. Field Trial Results}

The results presented in this section are from a single marine monitoring session, in which our team of robots operated within a $150 \mathrm{~m} \times 100 \mathrm{~m}$ coral reef region. Although this operational area may be considered small according to some marine monitoring standards, the current range of our system is predominantly limited by the UAV's operational capabilities, and hence can be easily resolved by upgrading to more efficient and higher capacity batteries, or switching to a more capable aerial vehicle.

We recorded both visual data and state information from all three vehicles, to allow the team's performance to be benchmarked and improved upon. Two important metrics used in the evaluation of our field trials consist of the durations for each phase in the reef monitoring process, and the distances traversed by the three robots.

Our monitoring session began with the launch of the Unicorn UAV, which was directed to cover the entire reef region in a circular orbit. The UAV flew at speeds between $10 \mathrm{~m} / \mathrm{s}$ and $20 \mathrm{~m} / \mathrm{s}$ in moderately turbulent wind conditions, and carried out aerial coverage first at $100 \mathrm{~m}$ and subsequently at $50 \mathrm{~m}$, as shown in Fig. 7(a). Aerial footage from the UAV was being continuously streamed to the home base through a radio frequency channel, and subsequently relayed to our web server. Therefore, this allowed the remotelylocated biologist to inspect the entire reef region using aerial views at two different scales, within 15 minutes following the launch of the UAV.

The scientist identified 4 sites of interest for up-close inspection after analyzing the aerial views for 10 minutes, and issued the corresponding waypoint directives to the home base using the web interface. These directives were then transmitted to the UAV, which began re-broadcasting the commands repeatedly while orbiting above the ASV in its coverage of the reef region. The ASV received all four target locations, after 25 minutes since the launch of the UAV. After completing both of its tasks of providing aerial footage of the coral reef and relaying waypoint directives from the biologist, the UAV proceeded to land while delegating the rest of the monitoring workload to the other two robots.

The ASV was operating as a caching station and relay unit, by re-broadcasting the target locations that it had received from the UAV continuously until the AUV had confirmed their reception. Upon receiving the target locations, the AUV activated its GPS waypoint follower to visit them one-byone, navigating via the porpoising motion to each of the specified waypoints. Through the porpoising motion, the AUV's piecewise linear path incorporated both localization and forward motion. The resulting trajectory executed by the AUV is shown in Fig. 8.

The Aqua AUV autonomously carried out the remainder of 


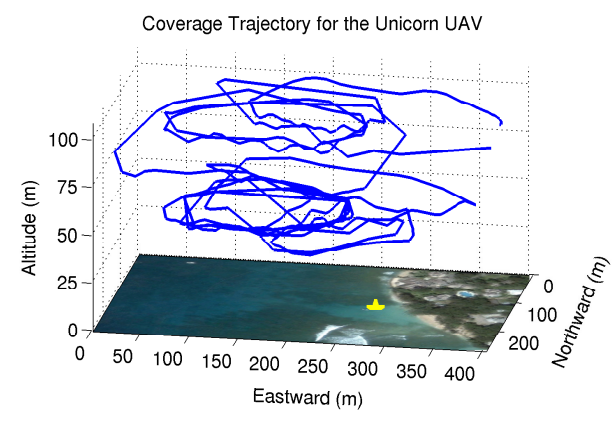

(a)

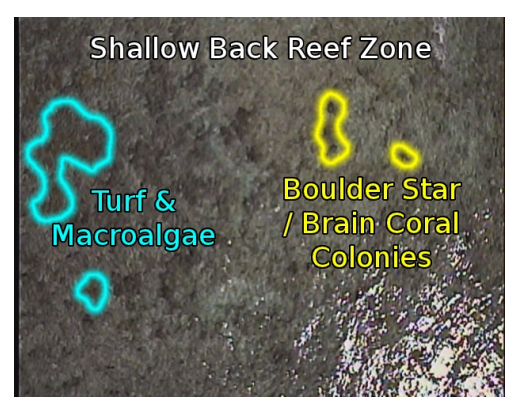

(b)

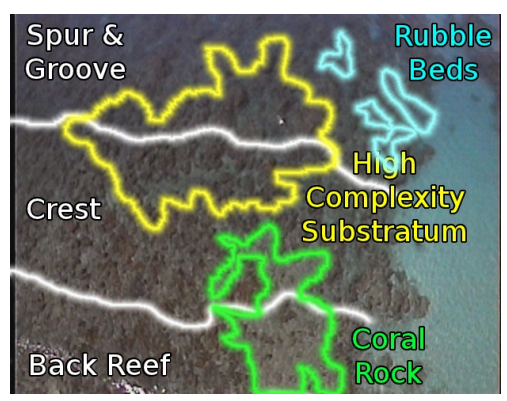

(c)

Fig. 7. (a) The Unicorn UAV carried out aerial coverage of a $150 \mathrm{~m} \times 100 \mathrm{~m}$ reef region at both $100 \mathrm{~m}$ and $50 \mathrm{~m}$ altitudes. Star icon depicts location of the MARE ASV. (b) The resulting images depict high resolution reef views, which enabled our expert biologist to identify diverse coral colonies and other areas of interest, and to estimate the relative depth of the reef, based on texture and color cues. (c) Frames acquired at $50 \mathrm{~m}$ provided close-up views while still being able to cover all three zones found on fringing reefs, namely spur and groove, crest, and back reef; the high complexity substratum area, characterized by strong variations in color and texture, is an especially interesting site to inspect from a biologist's perspective.

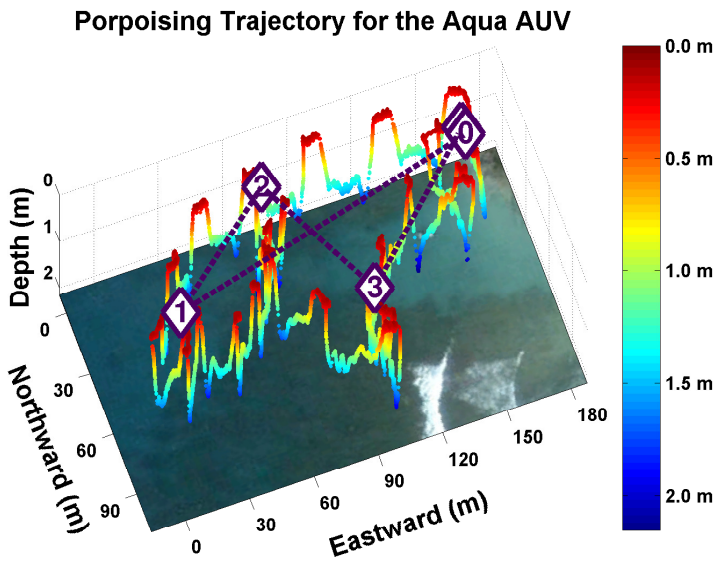

Fig. 8. Porpoising trajectory of the Aqua AUV.

the monitoring task, namely visiting all four target locations selected by the biologist, in about an hour. 20 minutes of that time were devoted to forward motion at about $0.6 \mathrm{~m} / \mathrm{s}$. The remaining 40 minutes were devoted to heaving up and down and GPS localization on the surface. As shown in Fig. 9 the AUV spent on average about $50 \mathrm{~s}$ at each porpoising stop to collect GPS readings with small spatial variance, and $40 \mathrm{~s}$ moving forward. We found that, although significant, this time interval was required to assure an accurate position estimate could be obtained.

As illustrated by Fig. 10(a), the porpoising trajectory of the Aqua robot did not exhibit straight line paths from one waypoint to the next. Fig. 10(b) shows that the cross track error of the GPS waypoint follower based on that motion was 13 meters on average. Deviations from the specified path are due to two factors: one is the presence of strong currents and waves, especially when the robot is heaving to the surface, and the other is the bearing correction based on the IMU yaw readings. The effects of these two factors on the resulting trajectory are independent and additive. This can be seen by considering the fact that each forward motion segment lasted the same amount of time, and thus noting that the variances in distances traveled between porpoising stops (red edges in Fig. 10(a)) is solely due to strong currents. In contrast, the heading errors and the resulting curvature of the trajectory is due to the drift of the IMU yaw, while the robot is pitching on the surface in order to obtain concentrated GPS readings. Despite these path deviations, the Aqua AUV reached each of the target locations at the accuracy of commercial GPS at $<$ $5 \mathrm{~m}$, which was suffient for it to capture underwater footage of the designated coral patches. The AUV returned to the initial location of the ASV 90 minutes after the launch of the UAV, at which point the experiment ended successfully, with the entire session lasting for two and half hours.

\section{CONCLUSION}

We have presented a multi-robot system that assists scientists in performing repeatable and consistent environmental monitoring. This type of monitoring enables scientists to study the evolution of the health of marine ecosystems. In particular, our goal is to facilitate the automation of coral reef inspection, by shifting the role of marine scientists from arduous manual data collection to the high-level identification of sites that need to be inspected.

Our robot team consisted of three vehicles that operated in different domains, namely an aerial (UAV), a surface (ASV), and an underwater vehicle (AUV). Given its hierarchical structure, this team was able to obtain multi-scale imagery at specified sites of interest efficiently. In particular, the UAV was able to quickly cover areas at a broad scale, which allowed the AUV to perform up-close inspection of sites with suspected high biodiversity only. The identification of such sites was performed by scientists who received the UAV imagery via the web. These scientists then issued high-level commands to the robot team, which subsequently carried out these commands completely autonomously.

We conducted a set of coral reef monitoring field trials involving this robot team and a remotely-located marine biologist, despite a multitude of environment- and vehiclespecific constraints. The experiments were performed over a $150 \mathrm{~m} \times 100 \mathrm{~m}$ region, which would have otherwise required multiple days of work for a marine scientist to manually inspect. Our team of robots carried out the reef monitoring task within two and half hours, which included an hourlong setup phase. We have thus demonstrated through these 


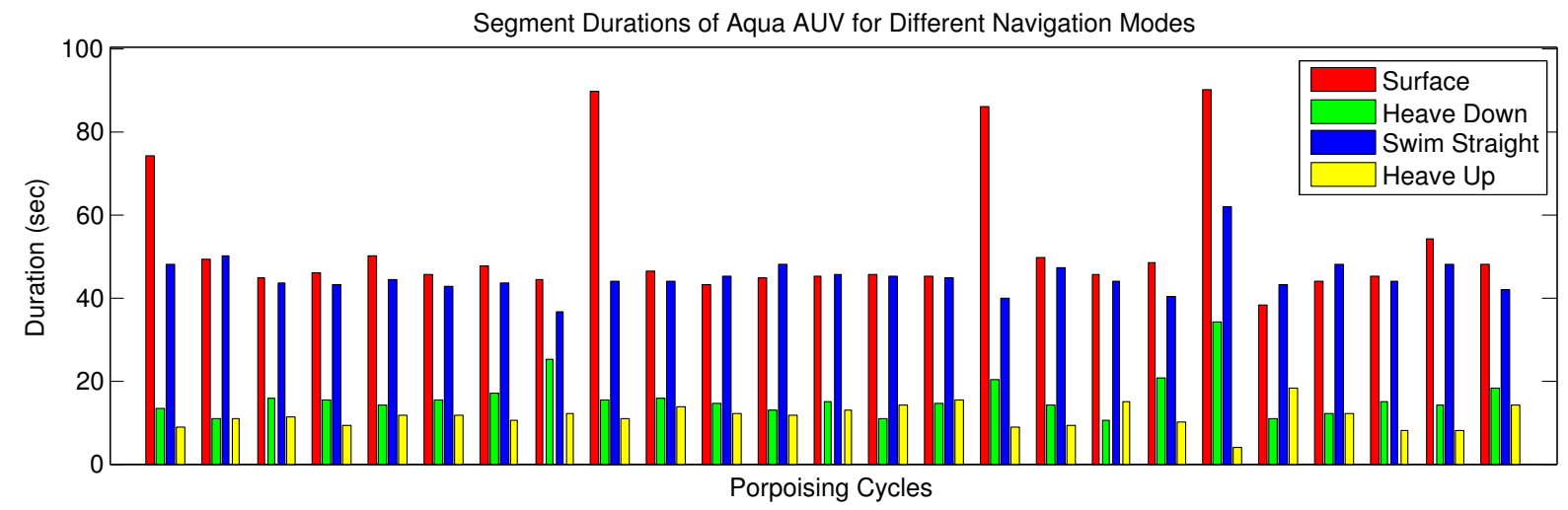

Fig. 9. Durations for each of porpoising modes during a navigation session for the Aqua AUV.

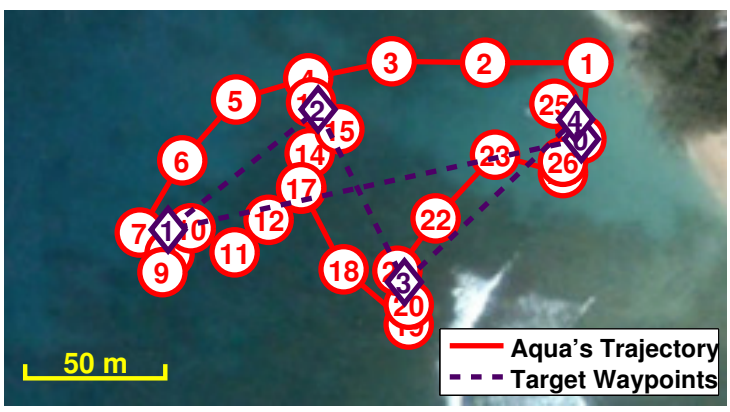

(a)

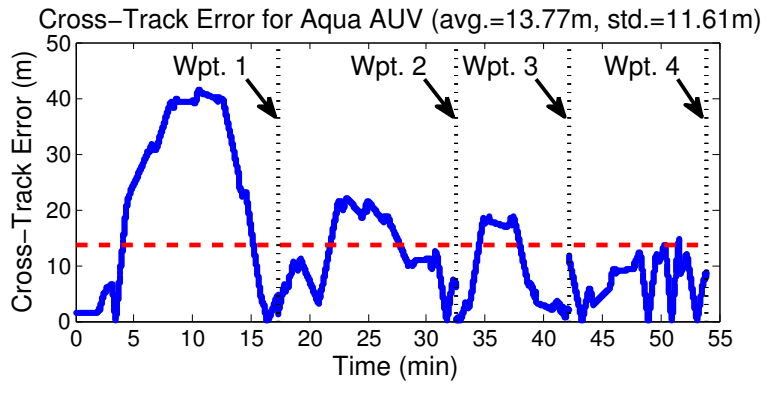

(b)

Fig. 10. (a) Overhead view of the Aqua AUV's trajectory. Diamond icons depict target locations suggested by the biologist for further inspection. Circular icons denote porpoising ascents during this session. (b) The crosstrack error for Aqua's trajectory suggests that it was pushed off-course due to strong currents.

field trials that our heterogeneous multi-robot system was capable of drastically improving the reef monitoring process compared to conventional diver-based approaches.

Future work for this system includes enabling the multirobot team to modify its monitoring plan according to environmental conditions, such as winds, waves, currents and other factors which are unfavorable to the control of the robots. We also aim to expand the coverage of target sites by gathering shots from additional scales, e.g. more altitudes for the UAV and more depths for the AUV. Finally, we are working to incorporate active monitoring roles into the MARE ASV, to work in tandem with the Aqua AUV.

\section{REFERENCES}

[1] M. Mulhall, "Saving the rainforests of the sea: An analysis of international efforts to conserve coral reefs," Duke Environmental Law and Policy Forum, vol. 19, pp. 321-351, 2007.

[2] G. Hodgson and J. Liebeler, The Global Coral Reef Crisis: Trends and Solutions. Reef Check Foundation, 2002.

[3] C. Rogers, et al., "Coral reef monitoring manual for the caribbean and western atlantic," Virgin Islands National Park, 110 p. Ilus., 1994.

[4] P. Valdivia y Alvarado, et al., "A coastal distributed autonomous sensor network," in OCEANS, 2011

[5] P. Sujit, J. Sousa, and F. Pereira, "UAV and AUVs coordination for ocean exploration," in OCEANS, 2009, pp. 1-7.

[6] A. Shafer, M. Benjamin, J. Leonard, and J. Curcio, "Autonomous cooperation of heterogeneous platforms for sea-based search tasks," in OCEANS, 2008, pp. 1-10.

[7] M. Dunbabin, P. Corke, I. Vasilescu, and D. Rus, "Data muling over underwater wireless sensor networks using an autonomous underwater vehicle," in Proc. of the IEEE Int. Conf. on Robotics and Automation, 2006, pp. 2091-2098.

[8] M. A. Hsieh, et al., "Adaptive teams of autonomous aerial and ground robots for situational awareness: Field reports," J. Field Robotics, vol. 24, no. 11-12, pp. 991-1014, 2007.

[9] J. Das, et al., "Simultaneous Tracking and Sampling of Dynamic Oceanographic Features with Autonomous Underwater Vehicles and Lagrangian Drifters," in Int. Symp. on Experimental Robotics, New Delhi, India, 2010.

[10] G. Podnar, et al., "Operation of robotic science boats using the telesupervised adaptive ocean sensor fleet system," in Proc. of the IEEE Int. Conf. on Robotics and Automation, 2008, pp. 1061-1068.

[11] N. Patrikalakis, et al., "Infrastructure for mobile sensor network in the singapore coastal zone," in Proc. of the Int. Society of Offshore and Polar Engineers, 2010.

[12] I. Rekleitis, et al., "Telepresence across the ocean," in Proc. of Conference on Computer and Robot Vision, 2010, pp. 261-268.

[13] Y. Girdhar, et al., "MARE: Marine Autonomous Robotic Explorer," in Proc. of the IEEE/RSJ Int. Conf. on Intelligent Robots and Systems, San Francisco, USA, 2011, pp. 5048-5053.

[14] G. Dudek, et al., "A visually guided swimming robot," in Proc. of the IEEE/RSJ Int. Conf. on Intelligent Robots and Systems, Edmonton, Alberta, Canada, August 2005, pp. 3604-3609.

[15] P. Giguere, G. Dudek, and C. Prahacs, "Characterization and modeling of rotational responses for an oscillating foil underwater robot," in Proc. of the IEEE/RSJ Int. Conf. on Intelligent Robots and Systems, 2006, pp. 3000-3005.

[16] J. Sattar, P. Giguere, and G. Dudek, "Sensor-based behavior control for an autonomous underwater vehicle," Int. J. Robotic Research, vol. 28 , no. 6, pp. 701-713, 2009.

[17] G. Dudek, J. Sattar, and A. Xu, "A visual language for robot control and programming: A human-interface study," in IEEE International Conference on Robotics and Automation, Rome, Lazio, Italy, April 2007, pp. 2507-2513.

[18] I. Poupyrev, H. Kato, and M. Billinghurst, ARToolKit User Manual Version 2.33, Human Interface Technology Lab, University of Washington, Seattle, Washington, 2000.

[19] A. Xu, C. Viriyasuthee, and I. Rekleitis, "Optimal complete terrain coverage using an unmanned aerial vehicle," in Proc. of the IEEE Int. Conf. on Robotics and Automation, Shanghai, China, May 2011, pp. 2513-2519. 